
allemande

48-2 | 2016

Les espaces publics des pays germanophones, des espaces publics transnationaux?

\title{
Le journal Arbeiterstimme, un espace public germanophone dans la Pologne de 1956 ?
}

\section{Pascal Fagot}

\section{OpenEdition}

\section{Journals}

Édition électronique

URL : https://journals.openedition.org/allemagne/428

DOI : 10.4000 /allemagne.428

ISSN : 2605-7913

Éditeur

Société d'études allemandes

Édition imprimée

Date de publication : 28 décembre 2016

Pagination : 345-356

ISSN : 0035-0974

Référence électronique

Pascal Fagot, «Le journal Arbeiterstimme, un espace public germanophone dans la Pologne de 1956 ? », Revue d'Allemagne et des pays de langue allemande [En ligne], 48-2 | 2016, mis en ligne le 28 décembre 2017, consulté le 20 mai 2021. URL : http://journals.openedition.org/allemagne/428 ; DOI : https://doi.org/10.4000/allemagne.428 


\section{Le journal Arbeiterstimme, un espace public germanophone dans la Pologne de 1956?}

\section{- Pascal Fagot*}

Staline venait tout juste de décéder quand, en juin 1953, après avoir protesté contre les augmentations des rythmes de travail, les ouvriers de la Stalinallee à Berlin-Est réclamèrent pour la RDA des réformes politiques. Trois ans plus tard, en 1956, dans un discours secret rapidement rendu public, Khrouchtchev dénonçait le stalinisme comme système dictatorial et criminel.

La même année, les Polonais et les Hongrois proclamaient à voix haute leur profonde insatisfaction quant à leur situation économique et politique. Mais tandis que le mouvement de protestation hongrois était violemment réprimé par les chars de l'Union soviétique, les Polonais parvenaient à imposer le retour de Władysław Gomułka à la direction du parti communiste, dont il avait été chassé en 1948 pour «déviationnisme " nationaliste.

Quand, dans un discours resté célèbre, Władysław Gomułka s’adressa le 25 février 1956 à la population de Varsovie ${ }^{(1)}$, ils étaient nombreux parmi le public à croire que la Pologne pourrait recouvrer la plus grande partie de son indépendance, à espérer que, dans le contexte donné, elle reprendrait son histoire en mains et s'inventerait un type de communisme dont elle pourrait au moins choisir la forme. On voulait édifier un État qui, tout en étant socialiste, n'en serait pas moins le lieu d'une démocratie qu'on dirait polonaise et qui serait fondée sur la liberté de pensée et d'expression. Cinquante années plus tard, le philosophe Leszek Kołakowski se souvient:

«En juin et en octobre [1956], le parti élut, malgré l’opposition de Moscou et dans une atmosphère d'enthousiasme populaire général, un nouveau dirigeant qui, comme beaucoup de gens le croyaient à tort, rétablirait l'ordre démocratique et même la souveraineté du pays » ${ }^{(2)}$.

* Professeur à l'Université de Strasbourg, EA 1341 Études germaniques.

1 https://www.youtube.com/watch?v=pwQTHsMSDF8 (23.06.2016).

2 Georges Mink, Marc Lazat, Mariusz J. Sielski (éd.), 1956, une date européenne, Lausanne, Noir sur Blanc, 2010, p. 148. 
Dans cette Pologne mouvementée et protestataire de 1956, la population germanophone était encore nombreuse. On estime généralement à douze millions le nombre des Allemandes et Allemands qui, dans des conditions souvent très difficiles, avaient, après 1945, dû rejoindre l'Allemagne dans ses nouvelles frontières ${ }^{(3)}$, mais, selon les sources et la façon dont on définissait la population allemande, ils étaient encore entre 120000 et un million qui continuaient de vivre en République populaire de Pologne ${ }^{(4)}$. Les uns avaient été contraints d'y demeurer parce qu'ils disposaient de compétences professionnelles indispensables à la remise en marche de l'économie polonaise, les autres avaient réussi à échapper à l'expulsion pour vivre dans ce qu'ils voulaient continuer de considérer comme leur Heimat, mais ils se demandaient maintenant, en 1956, s'ils avaient autrefois fait le bon choix. Ils savaient en effet que l'Allemagne occidentale avait entamé un miracle économique dont la Pologne ne pouvait que rêver, et ils avaient appris que, indépendamment de ce qu'ils avaient acquis ou non la citoyenneté polonaise, ils seraient encore longtemps perçus et traités comme les anciens citoyens d'un État vaincu et détesté.

Avant d'aborder le problème que nous voulons traiter ici de l'existence d'un «espace public » germanophone dans la Pologne de 1956, nous définirons avec Bernhard Peters l'espace public comme «sphère dans laquelle les citoyens d'une communauté politique démocratique réfléchissent librement et publiquement à la façon dont ils veulent régler les affaires publiques" dans un esprit de discussion, d'échange intellectuel et de respect mutuel ${ }^{(5)}$. Mais nous savons bien que, au-delà de cette définition dont les forums publiés sur Internet nous révèlent à quel point elle est utopique, les débats et discussions politiques donnent souvent lieu à de vifs conflits et controverses - Nancy Fraser parle $\mathrm{d}^{\text {" }}$ arène discursive " ${ }^{(6)}$-, aussi, nous complèterons cette conception de l'espace public par une remarque de Nilüfer Göle, qui explique en juin 2014 :

«Comme au théâtre, l'espace public représente le lieu où les différents acteurs, les nouveaux arrivés apparaissent sur scène et apprennent à jouer une pièce ensemble; ils se retrouvent dans le consensus, mais aussi dans la confrontation [...]. La sphère publique, comme le théâtre,

3 Cf. Klaus J. Bade, Pieter C. Emmer, Leo Lucassen, Jochen Oltmer, Enzyklopädie. Migration in Europa vom 17. Jahrhundert bis zur Gegenwart, Munich, Wilhelm Fink, 2010, p. 158: environ 18000000 d'Allemands vivaient avant 1939 dans les régions du Reich allemand intégrées après 1945 dans les territoires de la Pologne et de l’Union soviétique («Reichsdeutsche») et en dehors des frontières du Reich ( Volksdeutsche»). En ce qui concerne ce deuxième groupe, le chiffre varie selon que le critère retenu est celui de la nationalité (langue et culture) ou de la citoyenneté et a fait l'objet de controverses et contestations, notamment de la part de la Pologne. Parmi cette population dite allemande, 12000000 auraient fui ou auraient été expulsés vers l'Allemagne dans ses nouvelles frontières de 1945 (République fédérale d'Allemagne et République démocratique allemande), 500000 vivraient en Autriche et dans d'autres États, dont la Pologne - ce sont ces derniers qui nous occupent ici.

4 Dariusz Stola, Kraj bez wyścia? Migracje z Polski 1949-1989, Varsovie, Instytut Pamięci Narodowej, 2010, p. 67.

5 Bernhard Peters, Der Sinn von Öffentlichkeit, Francfort-sur-le-Main, Suhrkamp Taschenbuch, 2007, p. 69: «Sphäre öffentlicher, ungezwungener Meinungs- und Willensbildung der Mitglieder einer demokratischen politischen Gemeinschaft über die Regelung der öffentlichen Angelegenheiten », "Gleichheit und Reziprozität», "Offenheit für Themen und Beiträge», «diskursive Struktur der Kommunikation ».

6 Nancy Fraser, «Repenser la sphère publique: une contribution à la critique de la démocratie telle qu'elle existe réellement», CNRS, Hermes, (31) 2001, http://documents.irevues.inist.fr/bitstream/ handle/2042/14548/HERMES_2001_31_125.pdf;jsessionid=1B5E23A04FA5A3B4241DA0511C78FF2 5 ? sequence $=1(23.06 .2016)$. 
n'est pas un lieu purement discursif et rationnel où se confronteraient les opinions et les mots pour arriver à un consensus. C'est un lieu où se déroule un drame social, imprévisible, qui met en scène les singularités des corps et le pluralisme des perceptions du monde » ${ }^{(7)}$.

Aux antipodes de ces définitions de l'espace public, le stalinisme pratiquait au début des années 1950 une parole autoritaire qui, en imposant un consensus, ruinait toute possibilité d'espace public et que Bakhtine définit de la façon suivante:

«La parole autoritaire exige de nous d'être reconnue et assimilée, elle s'impose à nous, indépendamment de son degré de persuasion intérieure à notre égard; nous la trouvons comme déjà unie à ce qui fait autorité. [...] C'est une parole trouvée par avance, qu'on n’a pas à choisir parmi des paroles équivalentes. [...] La parole autoritaire exige de nous d'être reconnue inconditionnellement, et, non maîtrisée, assimilée librement avec nos mots à nous. [...] Elle pénètre dans notre conscience verbale telle une masse compacte et indivisible, il faut l'accepter tout entière ou tout entière la rejeter. Elle s'est inséparablement soudée à l'autorité (pouvoir politique, institution, personnalité) : avec elle, elle dure, avec elle, elle tombe ${ }^{(8)}$.

À la suite de la mort de Staline et avec la remise en question du régime politique qu'il avait édifié, la parole s'est libérée dans la Pologne de 1956 pour faire un temps place à une «décentralisation verbale et idéologique ${ }^{(9)}$ permettant la confrontation des discours et la constitution d'un espace public conçu comme lieu de rencontre et de confrontation des paroles et des idées et comme possibilité de contestation du pouvoir en place.

Le 18 octobre 1956, le magazine Die Zeit rapporte que, dans le nouveau contexte politique que s'étaient créé les Polonais, un important journal de Varsovie (Życie Warszawy) s'intéressait particulièrement et de façon critique à l'égard de la réalité politique et sociale au sort des Allemands de Pologne ${ }^{(10)}$. La question se pose pour nous de savoir dans quelle mesure cette population qui jusqu'au début des années 1950 était vouée à plus ou moins long terme à être expulsée et n’avait pas le statut officiel de minorité, a pu participer aux débats qui ont animé cet espace public polonais dont nous savons maintenant qu'il fut très éphémère, dans quelle mesure une ou des paroles allemandes ont pu se mêler et se confronter aux diverses paroles polonaises qui se sont alors fait entendre.

Pour répondre à cette question, nous allons feuilleter les pages du journal Arbeiterstimme, édité en allemand - évidemment sous contrôle du pouvoir polonais et avec la participation de nombreux contributeurs polonais -, entre 1951 et 1958 en Basse-Silésie polonaise, d'abord à Wałbrzych (Waldenburg), puis à Wrocław (Breslau), dont le tirage hebdomadaire puis quotidien à partir de 1955 adressait ses 30000 exemplaires à un lectorat germanophone essentiellement ouvrier et paysan.

Après nous être demandé dans un premier temps dans quelle mesure ce journal, qui se qualifiait lui-même de sozial-politische Tageszeitung et portait en exergue le célèbre «Proletarier aller Länder, vereinigt euch!» ${ }^{(11)}$, peut être considéré comme vecteur de la contestation polonaise auprès de la population germanophone de Pologne, nous

7 Nilüfer Göle, «La visibilité disruptive de l'Islam dans l'espace public européen: enjeux politiques, questions théoriques", http://www.sens-public.org/spip.php?article1085, juin 2014 (23.06.2016).

8 Mikhaïl Baкhtine, Esthétique et théorie du roman, Paris, Collection Tel Gallimard, 1978, p. 161.

9 Ibid., p. 186.

10 http://www.zeit.de/1956/42/die-deutschen-in-polen (23.06.2016).

11 «Prolétaires de tous les pays, unissez-vous!» 
nous interrogerons sur la façon dont il a participé de la constitution d'une "arène discursive» dans laquelle auraient pu débattre Allemands et Polonais. Nous verrons dans un dernier temps que la restauration rapide d'un monologisme autoritaire par les nouveaux gouvernants polonais et l'émigration concomitante de la population germanophone vers la RFA mirent ensemble fin à cette ébauche d'espace public germanopolonais qu'avait pu brièvement offrir le journal Arbeiterstimme.

\section{Le journal Arbeiterstimme comme porte-voix de la contestation polonaise}

Dès sa fondation, l'objectif du journal Arbeiterstimme avait été de fournir à la population germanophone de Pologne des informations en langue allemande sur la vie culturelle et politique de la nouvelle République populaire et de la gagner à la cause du socialisme; dans le contexte du stalinisme, il avait donc ouvert ses colonnes à l'idéologie officielle du pays et en avait exploité tout l'appareil rhétorique. Au moment où le gouvernement polonais était remis en question sous la pression des manifestations publiques, c'est souvent dans la langue qu'ils avaient appris à manier que les collaborateurs du journal expliquaient les changements politiques en cours. Après avoir reproduit en langue allemande l'intégralité du long discours de Władysław Gomułka du 24 octobre 1956 dans lequel il annonçait à la population varsovienne sa volonté de renouveler le socialisme ${ }^{(12)}$, c'est à l'aide de titres très semblables à ceux auxquels ils avaient habitué leurs lecteurs et affirmant l'adhésion de la population à la politique du parti au pouvoir qu'ils constataient l'accord parfait entre travailleurs allemands et polonais: «Die deutschen Arbeiter Schulter an Schulter mit den polnischen Werktätigen», ou encore: "die Werktätigen von Wałbrzych akzeptieren die Beschlüsse des VIII. Plenums der PZPR» ${ }^{(13)}$.

En réalité, bien qu'usant de la rhétorique habituelle et jusque-là obligatoire, le journal s'efforçait de faire comprendre à son lectorat allemand le bouleversement politique que connaissait alors la Pologne, où l'on réclamait tout à la fois une réforme profonde du socialisme et revendiquait son autonomie à l'égard de l'Union soviétique.

Par-delà le discours convenu du stalinisme, nous notons en effet certains renouvellements stylistiques traduisant le fait que Arbeiterstimme participait de cet enthousiasme quasi-révolutionnaire dont Kołakowski se souviendra cinquante années plus tard. C'est ainsi qu'en novembre 1956, le journaliste polonais M. Gruszkiewicz racontait avec émotion les événements qu'il venait de vivre:

«Wir erwachten eines Tages und sahen auf einmal die Welt mit anderen Augen an. Dinge, die bis vor kurzem für uns eine Selbstverständlichkeit waren, hörten auf, selbstverständlich zu sein, gewohnte Worte klangen leer und falsch, Begriffe bekamen einen neuen Inhalt. Anders sahen wir die Vergangenheit, und anders die Gegenwart. Wir analysierten, zogen Schlüsse und begannen, die Dinge bei ihrem Namen zu nennen ${ }^{(14)}$.

12 Voir note 1.

13 Arbeiterstimme (désormais $A S$ ), 577: «Les travailleurs allemands aux côtés des travailleurs polonais»; «Les travailleurs de Wałbrzych acceptent les décisions du VIII ${ }^{e}$ Plenum du PZPR» (PZPR: Polska Zjednoczona Partia Robotnicza /Parti Ouvrier Unifié Polonais).

$14 A S, 595$ : «Un jour, nous nous sommes éveillés et avons subitement vu le monde avec d'autres yeux. Ce qui, il y a peu, nous semblait évident, cessa d'être évident; les paroles auxquelles nous étions habitués sonnaient creux et faux, les concepts avaient un autre contenu. Nous vîmes autrement le passé et 
Pour donner à son lectorat germanophone accès aux discussions et contestations qui animaient alors l'espace public polonais, le journal Arbeiterstimme lui présentait dans sa langue ce nouveau regard que les Polonais portaient sur leur réalité. Dès octobre 1956, il lui expliquait la remise en question fondamentale du système existant:

«Der Stalinismus bedeutet nicht allein Gewalt, welche der Wirtschaft angetan wurde, sondern auch Gewalt, die selbst den Menschen gegenüber ständig angewandt wurde, [...] er bedeutet ein System des Terrors, der Bespitzelung und der Provokation, sowie des brutalen Drucks zwecks Erlangung des Anscheins einer "moralisch" politischen Einheit des Volkes » ${ }^{(15)}$.

C'est également dans cet état d'esprit que le journal éditait une série d'articles intitulée «Für oder gegen den Sozialismus» (Pour ou contre le socialisme) qui étaient la traduction de textes extraits du journal étudiant réformiste Po Prostu ${ }^{(16)}$ et, par exemple, exposaient les principes de la nouvelle autogestion ouvrière (Arbeiterselbstverwaltung $)^{(17)}$.

En cette fin d'année 1956, Arbeiterstimme ne jouait pas seulement le rôle d'intermédiaire entre un espace public polonais en pleine ébullition et une population germanophone qui, souvent, ne se sentait pas concernée par les problèmes qui y étaient discutés, il s'efforçait de convaincre la population allemande de participer à cet espace public en gestation et de contribuer à un renouveau politique qui devrait lui permettre de faire valoir ses intérêts propres. En novembre 1956, dans un article intitulé «Kumpel, auch dir gehört das Wort!», on exposait au lecteur allemand les nouvelles possibilités qui lui étaient offertes de participer à la vie de l'entreprise:

«- Wer soll mitmachen?

- Alle, alle, alle.

— Wir Deutschen auch? fällt plötzlich eine Frage während einer Versammlung.

- Selbstverständlich!

— Wir verstehen aber nichts davon. Man spricht nur Polnisch [...]

— Eure Schuld! Jede Grube besitzt ihren deutschen Arbeiterrat» ${ }^{(18)}$.

\section{Arbeiterstimme comme porte-voix de la contestation des Allemands de Pologne}

Les Allemands de Pologne entendirent le message et comprirent le parti qu'ils pourraient en tirer. Dans un mouvement de retour, ils utilisèrent Arbeiterstimme comme nouvel espace public de discussion en Pologne. Tandis que le journal avait jusqu'alors permis aux Polonais de s'adresser à la population germanophone de Pologne, c'était

autrement le présent. Nous fîmes des analyses, nous tirâmes des conclusions et nous commençâmes à appeler les choses par leur nom.»

$15 A S, 590$ : «Le stalinisme ne signifie pas seulement la violence faite à l'économie, mais aussi la violence constamment utilisée à l'encontre des personnes elles-mêmes [...], il signifie un système de terreur, d'espionnage et de provocation, ainsi que de pression brutale dont l'objectif était de créer l'apparence d'une unité morale et politique du peuple.»

16 Le journal Po Prostu, qui tire en 1956 à 150000 exemplaires, et qui se définit comme «Hebdomadaire des étudiants et des jeunes intellectuels» (Tygodnik Studentów i Młodej Inteligencji) est en 1956 un acteur essentiel de la contestation politique. Il sera fermé en 1957.

17 AS, 567.

$18 A S, 596$ : «Qui doit participer? - Tous, tous, tous. Pendant une réunion, quelqu'un pose tout à coup la question: Nous aussi, les Allemands? - Évidemment! - Mais nous n’y comprenons rien. On ne parle que polonais $[. .$.$] - C'est votre faute! Chaque mine a son conseil des travailleurs allemands. »$ 
maintenant cette dernière qui, par l'intermédiaire du même journal, lançait ses idées dans l'espace public de la République populaire de Pologne.

Prenons pour exemple une délégation réunissant des Allemands membres du parti communiste, de l'organisation de jeunesse et du syndicat polonais qui, en novembre 1956, se rendit à Varsovie pour présenter aux autorités centrales ses récriminations et revendications.

Ce groupe, dont l'intervention était reproduite dans Arbeiterstimme, prenait la parole au nom des Allemands de Pologne pour exposer tout d'abord leur profonde insatisfaction quant à la façon dont ils étaient traités :

«Leider zeigen die örtlichen Behörden gegenüber den dringendsten Beschwerden nicht immer das notwendige Interesse, so daß die dadurch auftretende Unzufriedenheit und Verärgerung innerhalb der deutschen Bevölkerung gegenwärtig den Höhepunkt erreicht hat [...]. [Es] muß offen gesagt werden, daß der deutsche Arbeiter innerhalb der Arbeitsbetriebe, wie auch bei den Behörden, aufgrund seiner mangelhaften Kenntnisse der polnischen Sprache, nicht immer sein laut Verfassung der Volksrepublik Polen zugebilligtes Recht erhielt » ${ }^{(19)}$.

Il posait ensuite ses revendications, notamment le droit au «rapatriement» en Allemagne et la reconnaissance officielle que la population allemande de Pologne constituait une minorité nationale:

«Keinem Bürger deutscher Nationalität, der von seinem Recht auf Aussiedlung Gebrauch machen will, darf daran gehindert werden [...]. Als beschämende Tatsache muß festgestellt werden, daß die Regierung der Deutschen Demokratischen Republik als sozialistischer und mit Volkspolen befreundeter Staat bisher keine Schritte zur Anerkennung der Bürger deutscher Nationalität als nationale Minderheit in Volkspolen unternommen hat. Es ist daher leicht verständlich, daß sich die Sympathie eines Teiles der deutschen Minderheit in Volkspolen dem kapitalistischen Lager, das heißt Westdeutschland, zugewandt hat, welches diesen Faktor zu Hetz- und Propagandaaktionen ausnützt» ${ }^{(20)}$.

Dans la même veine, des enseignants des écoles germanophones de Silésie présentaient le 27 octobre 1956, trois jours après le discours de Gomułka à Varsovie, des doléances qui dépassaient largement le cadre de leurs compétences professionnelles:

«Die Lehrer der deutschsprachigen Schulen der Woiwodschaft Wrocław erwarten, daß das ZK der PZPR in Verbindung mit der Regierung der Volksrepublik Polen versucht, das Problem der deutschen Bevölkerung in Polen in seiner Gesamtheit zu lösen, insbesondere, daß :

19 AS, 606: "Malheureusement, les autorités locales ne s’intéressent pas toujours comme elles le devraient aux plaintes les plus urgentes, de sorte que, dans la population allemande, l'insatisfaction et la colère qui en résultent atteignent actuellement leur point culminant. [...] Il faut dire ouvertement que, en raison de sa connaissance insuffisante de la langue polonaise, le travailleur allemand ne jouit pas toujours dans les entreprises comme dans les administrations des droits que leur reconnaît la constitution de la République populaire de Pologne.»

20 Ibid.: "Aucun citoyen de nationalité allemande désireux d'user de son droit à l'émigration ne peut en être empêché. [...] Il faut qualifier de honteux le fait que, en tant qu'État socialiste ami de la Pologne populaire, le gouvernement de la République démocratique allemande n'a encore entrepris aucune démarche pour obtenir la reconnaissance des citoyens de nationalité allemande comme minorité allemande en Pologne populaire. Il est donc tout à fait compréhensible que la sympathie d'une partie de la minorité allemande de Pologne populaire se soit tournée vers le camp capitaliste, c'est-à-dire vers l'Allemagne de l'Ouest, qui exploite cet état de fait dans ses actions de dénigrement et de propagande.» 
1) allen Einwohnern deutscher Nationalität, die von ihrem Recht auf Repatriierung in ihr deutsches Vaterland Gebrauch machen wollen, die Möglichkeit der Rückkehr nach Deutschland gegeben wird,

2) allen denjenigen, die in der Volksrepublik Polen bleiben wollen, alle Möglichkeit der kulturellen und gesellschaftlichen Betätigung gewährt werden [...]» ${ }^{(21)}$.

Ce qui est remarquable est que, en cette fin d'année 1956, les représentants des autorités polonaises acceptaient de répondre aux critiques que leur avaient présentées les représentants de la population allemande de Pologne. Dans les mêmes colonnes de Arbeiterstimme, ils promettaient une remise en question fondamentale de la politique menée jusqu'alors à l'égard de la population allemande:

«Bei der Aussiedlung [der deutschen Bevölkerung] wurden viele Fehler begangen. So manchem Menschen wurde Unrecht zugefügt. In vielen Fällen wandte man den Grundsatz der kollektiven Verantwortung an, was im Widerspruch zum sozialistischen Humanismus steht [...] Fällen, in denen man auf die Würde des Menschen keinerlei Rücksicht nahm. [...] es ist jedoch erforderlich, mit den Fehlern der Vergangenheit abzurechnen. Diese Fehler entsprangen dem damaligen System und der Arbeit gewisser unverantwortlicher Menschen " ${ }^{(22)}$.

À ces paroles se joignaient celles d'intellectuels polonais qui, pratiquant la décentralisation du regard que Bakhtine définit comme fondement du dialogisme, considéraient l'histoire récente à partir de la perspective de la population allemande de Pologne.

Andrzej Wakar (1920-1995), jeune historien polonais, condamnait par exemple la politique nationaliste du parti communiste polonais et l'attitude de la population polonaise à l'égard des Masuriens, comme on appelle en Pologne la population allemande de l'ancienne Prusse Orientale:

«Keine Statistik ist imstande, zu erfassen, wie oft die Masuren schlecht behandelt wurden [...], wieviel Unrecht sie erlitten seitens des durch Nationalismus verseuchten zuziehenden Bevölkerung, wie oft sie von gewissenlosen Schwindlern [...] in dem Wirrwarr unserer Gesetze begaunert und ausgenutzt wurden. [...] Wir haben ein Klima geschaffen, in dem die Masuren nicht atmen können. [...] Der "Kampf um das Polentum" [...] ist ein ganzer Roman des Irrsinns und der Verbrechen [...] [eine] Maschine, die im Namen der "polnischen Staatsräson" die Seelen und Körper der Einheimischen zermalmte» ${ }^{(23)}$.

21 AS, 587: «Les enseignants des écoles germanophones de la Voïvodie de Wroclaw attendent que le Comité central du PZPR en relation avec le gouvernement de la République populaire de Pologne tente de résoudre le problème de la population allemande dans son ensemble et en particulier : 1) qu'il donne à tous les habitants de nationalité allemande qui veulent user de leur droit au rapatriement dans leur patrie allemande la possibilité de rentrer en Allemagne, 2) qu'il garantisse à tous ceux qui veulent rester en République populaire de Pologne la possibilité d'avoir des activités culturelles et sociales.»

22 Ibid.: «Lors du déplacement [de la population allemande vers l'Allemagne], de nombreuses erreurs ont été commises. Bien des individus ont subi des injustices. Dans de nombreux cas, on a appliqué le principe de la responsabilité collective, ce qui est en contradiction avec l'humanisme socialiste. [...] des cas dans lesquels on n'a tenu aucun compte de la dignité humaine. [...] il est pourtant nécessaire que nous regardions en face les fautes du passé. Ces fautes provenaient de l'ancien système et du travail de certaines personnes irresponsables.»

$23 A S$, 590: "Aucune statistique n'est en mesure de rendre compte du nombre de mauvais traitements dont les Masuriens ont été victimes, de la quantité d'injustices dont ils ont souffert de la part d'une population arrivante infestée par le nationalisme, de toutes les fois où, dans l'imbroglio de nos lois, ils ont été abusés et exploités par des voyous sans scrupules [...]. Nous avons créé un climat dans lequel les Masuriens ne peuvent pas respirer. [...] le combat pour la polonité [...] n'est qu'une longue suite de 
Vingt-cinq ans plus tard, en 1981, à un moment où, de nouveau, la contestation de la politique du parti communiste au pouvoir libéra la parole et remplit l'espace public polonais, Jan Józef Lipski, qui avait en 1956 collaboré au journal Po Prostu, publia un texte intitulé Deux patries, deux patriotismes. Considérations sur la mégalomanie nationale et la xénophobie des Polonais ${ }^{(24)}$. Dans ce texte, qui marqua son époque, il appelait les Polonais à reconsidérer le récit qu'ils faisaient de l'histoire de leur relation avec les Allemands. En réalité, il reprenait de grands traits de l'argumentation qu'avaient déjà développée certains intellectuels polonais dans les numéros de Arbeiterstimme de la fin de l'année 1956:

«Dans la conscience polonaise de nos relations historiques avec les Allemands s'est constituée une masse de mythes et d'idées fausses qu'au nom de la vérité et pour notre propre guérison, il faudra un jour dénoncer comme mensonges: des représentations fausses de notre propre histoire constituent une pathologie de l'âme de notre peuple, elles servent avant tout à nourrir la xénophobie et la mégalomanie nationale» ${ }^{(25)}$.

\section{La fin d'un espace de contestation et de discussion germano-polonais}

La période où le journal Arbeiterstimme faisait figure d'espace de discussion dans lequel pouvaient s'exprimer et se renforcer mutuellement les contestations allemandes et polonaises, dura peu de temps. Très rapidement, dès la fin de l'année 1956, le parti communiste réinstalla en Pologne le monologisme politique et autoritaire qui avait prévalu jusqu'alors; tout aussi rapidement, le journal lui emboîta le pas pour redevenir le porte-voix du gouvernement et de sa nouvelle propagande du succès assurant que les réformes nécessaires avaient été faites et qu'il fallait maintenant reprendre une vie normale. Dès décembre 1956, Arbeiterstimme invitait ses lecteurs à voter lors des élections législatives de janvier 1957 pour le Front d'unité nationale (Nationale Einheitsfront), qui reconstituait le consensus politique en rassemblant autour du parti communiste la plupart des partis politiques et organisations:

«Sie vereinigt in sich Millionen von Bürgern, Parteiangehörige, Parteilose, Vereinigte und Nichtvereinigte - alle die, die in täglicher schwerer Mühe an der Zukunft unseres Vaterlandes schmieden» ${ }^{(26)}$.

En avril 1958, le journal affirmait et confirmait officiellement son engagement aux côtés du parti : « [...] daß wir immer auf einer bestimmten Seite der Barrikade stehen » ${ }^{(27)}$.

folies et de crimes [...] [une] machine qui, au nom de la “raison d’État polonaise”, a broyé les âmes et les corps des autochtones.»

24 Dwie ojczyzny - dwa patriotyzmy. Uwagi o megalomanii i ksenofobii narodowej Polaków. Le texte original est en ligne à l'adresse: http://m.wyborcza.pl/wyborcza/1,132749,16932860,Dwie_ojczyzny_ dwa_patriotyzmy.html?disableRedirects=true (consulté le 15.9.2016).

25 Ibid.: «W polskiej świadomości naszych stosunków historycznych z Niemcami narosło masę mitów i fałszywych wyobrażeń, które trzeba będzie kiedyś odkłamać w imię prawdy i w celu leczenia siebie samych: fałszywe wyobrażenia o własnej historii są chorobą ducha narodu, służą przeważnie za pożywkę ksenofobii i megalomanii narodowej.»

26 AS, 621: «Il réunit en lui des millions de citoyens, des membres du parti, des sans-parti, des gens participant ou non à des associations - tous ceux qui, en un difficile effort quotidien, forgent l'avenir de notre patrie.»

27 AS, 1022-1023: «[...] que nous nous trouvons toujours d'un certain côté de la barricade». 
En ce qui concerne la population germanophone de Pologne, le message que lui transmettait Arbeiterstimme était de nouveau d'une clarté que ne venait plus troubler aucune volonté de contestation politique: le nouveau gouvernement de la Pologne populaire avait satisfait ou était en train de satisfaire à ses revendications, de sorte que, comme leurs conditions de vie ne cessaient de s'améliorer, les Allemands de Pologne n'avaient plus aucune raison sérieuse de protester ou de demander à quitter le pays.

En février 1958, Julian Bartosz, qui avait un temps dénoncé le non-respect des droits des Allemands de Pologne, expliquait maintenant dans un reportage réalisé à Świdnica, autrefois Schweidnitz:

«[die Deutschen in Polen] leben weiter ihr ruhiges und geordnetes Leben [...]. Alle Probleme, die vor zwei- drei Jahren das Leben der Deutschen beeinträchtigten und erschwerten, wurden beseitigt ${ }^{(28)}$.

On s'accordait pour expliquer que le changement de gouvernement avait permis de solutionner tous les problèmes auxquels avait autrefois été confrontée la population allemande de Pologne, que ce n'était donc pas aux Polonais ou à leur gouvernement qu'elle devait maintenant ses difficultés, mais au gouvernement dit «fasciste» de la République fédérale d'Allemagne et aux groupements d'expulsés qui y avaient été créés et dont le premier objectif était, pour leur malheur, de convaincre les Allemands de Pologne d'émigrer vers la RFA.

En 1957-1958, le journal avait pour premier objectif de dénoncer ce mouvement d'émigration massive vers l'Allemagne occidentale ${ }^{(29)}$. Il fallait prouver aux candidats au départ qu'ils avaient une fausse représentation de la République fédérale d'Allemagne et que, contrairement à ce qu'ils attendaient et espéraient, ils y seraient mal accueillis. Un correspondant ouest-allemand du journal expliquait à ses lecteurs:

«Ein Notschrei geht durch die Bundesrepublik, ein Notschrei, der kein Echo findet, der verhallt an den Massenlagern an den Zonengrenzen, [...] "schickt uns zurück nach Polen" flehen die Aussiedler, [...] "die Polen taten mehr für uns als die Brüder in Deutschland tun”, meint ein uraltes Mütterlein, “Gott wird sie strafen! Gott wird sie strafen!” » ${ }^{(30)}$.

Le journal débordait de récits illustrant le mauvais accueil fait en Allemagne occidentale aux «rapatriés» de Pologne et que l'historien allemand Andreas Kossert a récemment redécouvert dans son ouvrage intitulé Kalte Heimat ${ }^{(31)}$.

Pourtant, jusqu'en 1958, et malgré une reprise en mains énergique du journal Arbeiterstimme par les nouvelles autorités politiques, on y rencontrait des traces de ce qui

28 AS, 984: Les Allemands de Pologne «continuent de mener une vie tranquille et ordonnée [...] tous les problèmes qui, il y a deux ou trois ans, perturbaient ou rendaient leur vie difficile, ont été résolus».

29 Voir à ce sujet Pascal FAgot, «Le départ des Allemands de Pologne dans les années 1950, vu à travers des documents administratifs publiés en Pologne en 2010», in: Carola HäHNEL-MeSNARD, Dominique Herbet (dir.), Fuite et expulsion des Allemands. Transnationalité et représentations 19$21^{e}$ siècle, Villeneuve d'Ascq, Presses Universitaires du Septentrion, 2016, p. 103-114.

$30 A S, 972$ : «Un cri de détresse parcourt la République fédérale, un cri qui ne rencontre aucun écho, qui se perd dans les camps construits aux frontières de la zone [...] "renvoyez-nous en Pologne" conjurent les rapatriés; une très vieille femme déclare: "les Polonais ont fait davantage pour nous que ne le font nos frères en Allemagne, Dieu les punira!, Dieu les punira!"”

31 Andreas Kossert, Kalte Heimat, Die Geschichte der deutschen Vertriebenen nach 1945, Munich, Siedler Verlag, 2008. 
avait brièvement constitué un espace de protestation germano-polonaise ouvert à différents publics et à différentes voix.

Prenons pour exemple un long article de novembre 1957 qui, tout en expliquant et en justifiant la fermeture du journal étudiant Po Prostu, citait longuement ce dernier et offrait au lecteur un dernier accès direct à sa parole et à sa pensée. Un bref extrait de cet article suffit à illustrer ce propos surprenant dans un journal déclarant soutenir le parti et le gouvernement:

«Wäre es nicht angebracht, die Partei aufzulösen? Wäre es nicht besser, die Partei zu liquidieren und von neuem mit dem Aufbau einer kommunistischen Partei in Polen zu beginnen?» ${ }^{(32)}$.

C'est dans le courrier des lecteurs et dans la relation du journal avec ces derniers que l'on rencontre les plus nombreuses traces de l'espace public de contestation qu'avait brièvement été Arbeiterstimme.

En avril 1958, peu de temps avant que le journal ne cessât de paraître, un lecteur lui adressait une lettre publiée parmi le courrier des lecteurs dans laquelle il critiquait le nouveau ton et la nouvelle direction politique du quotidien, dont il affirmait qu'il n'était plus qu'un «journal polonais en langue allemande» («eine polnische Zeitung in deutscher Sprache») et même la «filiale d’un journal de Berlin-Est» («die Filiale eines Ostberliner Blattes»), et déclarait regretter la période qui avait suivi le mois d'octobre 1956:

«Ihre politische Marschroute liegt fest, zugestanden. In der Sache können Sie nicht viel anders schreiben, selbst wenn Sie es wollten, aber in der Form, im Ton, in der Musik, können sie eine andere Art finden. Sie konnten es jedenfalls in den Oktobertagen. Ich erinnere mich noch mit Vergnügen des frischen Luftzuges, der damals durch die Spalten Ihrer Zeitung ging» ${ }^{(33)}$.

En cette période où il n'existait aucune relation diplomatique entre la République populaire de Pologne et la République fédérale d'Allemagne, l'auteur de ce même texte proposait un changement radical dans la politique étrangère polonaise:

«Denken Sie daran, daß nichts verhängnisvoller wäre, die Bundesrepublik mit jenen Augen anzusehen, wie es der Funktionär der DDR tut. Die Bundesrepublik soll der Freund von morgen sein, da sie es heute noch nicht sein kann. Dieser Freundschaft mit der Bundesrepublik und dem gesamten deutschen Volk den Weg zu ebnen, ist, wie mir scheint, eine wichtige Aufgabe einer Zeitung in deutscher Sprache in Polen ${ }^{(34)}$.

Certes, ce texte était suivi d'un commentaire rectificatif dénonçant la militarisation de la République fédérale d'Allemagne et très conforme à la «feuille de route politique »

32 AS, 877: «Ne faudrait-il pas mieux dissoudre le parti? Ne serait-il pas mieux de liquider le parti et de recommencer à zéro en construisant un parti communiste en Pologne?»

33 AS, 1022-1023: «Votre feuille de route politique est définie, il faut le reconnaître. En ce qui concerne le contenu, vous ne pouvez pas changer grand-chose, même si vous le vouliez, mais dans la forme, dans le ton, dans la musique, vous pouvez faire preuve d'imagination. En tout cas, vous avez su le faire dans les jours d'octobre. Je me souviens encore avec plaisir de la fraîcheur du courant d'air qui soufflait alors sur les colonnes de votre journal.»

34 Ibid.: "Pensez qu'il n'y aurait rien de plus fatal que de regarder la République fédérale avec les yeux du fonctionnaire de RDA. La République fédérale doit être l'ami de demain, puisqu'elle ne peut pas l'être déjà aujourd'hui. Préparer la voie qui mène à cette amitié avec la République fédérale et avec l'ensemble du peuple allemand est, me semble-t-il, une mission importante pour un journal de langue allemande en Pologne.» 
du journal, mais le fait même qu'il ait été publié permettait de confronter directement le lecteur à d'autres voix et d'autres opinions que celles du parti au pouvoir.

Cette double fonction de porte-voix du gouvernement polonais et de la contestation de la population allemande se faisait également jour à travers le paradoxe que, d'une part, le journal exposait à son lectorat toutes les bonnes raisons qu'il avait de rester en Pologne et de ne pas émigrer vers l'Allemagne, tandis que, d'autre part, il l'aidait dans ses démarches en répondant très précisément aux questions relatives aux conditions matérielles du voyage vers l'Allemagne et aux formalités à entreprendre pour obtenir l'autorisation de quitter la Pologne.

\section{Conclusion}

En entrée de cet article, nous nous sommes posé la question de savoir si Arbeiterstimme, journal germanophone en Pologne qui paraît de 1951 à 1958 à un rythme d'abord hebdomadaire puis quotidien, pouvait être considéré comme un espace public germano-polonais en langue allemande.

Dans une première partie, nous avons constaté que jusqu'en 1956, à l'image de l'ensemble de la presse polonaise des années du stalinisme, ce journal germanophone avait été au service de la parole autoritaire du parti communiste polonais et avait usé d'une rhétorique très semblable à celle que l'on pouvait par exemple rencontrer dans les journaux de République démocratique allemande. Mais nous avons ensuite constaté le changement de ton et de contenu qu'avaient permis les événements polonais d'octobre 1956. À ce moment-là, Arbeiterstimme expliquait à son lectorat les débats qui animaient l'espace public polonais et jouait ainsi le rôle de porte-voix de la contestation politique polonaise.

La deuxième partie a expliqué que la population allemande de Pologne avait saisi l'opportunité que lui offraient les mouvements de contestation polonais pour faire de Arbeiterstimme un nouvel espace public lui permettant à la fois de discuter des sujets qui l'occupaient et de mettre ses revendications sur la place publique polonaise. De leur côté, des intellectuels polonais se rendaient dans ce nouvel espace pour participer aux débats, apporter leur soutien aux doléances allemandes et critiquer la politique menée jusqu'alors par le gouvernement polonais à l'égard de la population germanophone. Arbeiterstimme ne servait plus seulement à informer sur les débats qui se déroulaient dans l'espace public polonais, il devenait lui-même un espace de débats et de controverses dans lequel s'articulait une contestation germano-polonaise du pouvoir polonais.

La dernière partie de notre travail a finalement révélé que cet état de fait n’avait duré que très peu de temps. Dès la fin de l'année 1956, alors qu'il s'agissait de procéder à de nouvelles élections législatives, le pouvoir polonais avait rétabli le monologisme officiel sur lequel il avait fondé sa communication dans les dernières années. Arbeiterstimme ne lui avait pas fait défaut et avait repris la rhétorique consensuelle qu'il avait appris à si bien maîtriser et qui convenait si mal à un espace public digne de ce nom.

Pourtant, les années 1957 et 1958 portaient encore les traces de l’histoire récente sous la forme d'un double langage qui accompagna le journal jusqu'à ce qu'il cessât de paraître en avril 1958: le courrier des lecteurs, les longues citations de textes protestataires, le soutien à l'émigration de la population germanophone vers l'Allemagne, tous 
ces éléments continuèrent un temps de faire vivre la discussion et la contestation qui avaient pu s'exprimer à la fin de l'année 1956.

Mais ce n'étaient là que les derniers soubresauts de ce qui aurait pu devenir un espace public de débats germano-polonais. Tandis que le pouvoir polonais fermait ce lieu de discussion, la population germanophone de Pologne s'en détournait pour émigrer massivement vers la République fédérale d'Allemagne. Il faudra attendre les années 1980 et 1990 pour que, à la faveur de nouveaux bouleversements politiques en Pologne et d'une nouvelle libération de la parole, se reconstitue un espace public germano-polonais dans lequel pourraient reprendre la discussion sur l'histoire récente et les possibilités d'une autre relation germano-polonaise.

\section{Résumé}

À partir de l'analyse du quotidien germanophone Arbeiterstimme paraissant en 1956 à Wroclaw/Breslau, cet article s'interroge sur l'existence en Pologne d'un espace public dans lequel les Allemands de Pologne auraient pu joindre et confronter leur parole à celle des (con)citoyens polonais. Il parvient à la conclusion que si, à la fin de l'année 1956, le journal a effectivement servi de lieu d'expression de vives contestations allemandes et polonaises du pouvoir en place, il a très vite été repris en mains par les autorités politiques qui, jusqu'aux événements des années 1980/1990, ont mis fin à toute velléité de création d'un espace public germano-polonais.

\section{Zusammenfassung}

Anhand einer Untersuchung der deutschsprachigen in Wrocław/Breslau erscheinenden Tageszeitung Arbeiterstimme will der Artikel der Frage nach der Existenz einer Öffentlichkeit nachgehen, in der sich 1956 polnische und deutsche Stimmen artikuliert und konfrontiert hätten. Es stellt sich heraus, dass die Tageszeitung am Ende des Jahres 1956 tatsächlich als Ort eines heftigen deutschen und polnischen Protestes fungieren konnte, dass die regierende Partei jedoch schnell und bis 1980/1990 dieser streitenden deutsch-polnischen Öffentlichkeit ein Ende setzen konnte.

\section{Abstract}

Basing on the analysis of the daily Arbeiterstimme edited in 1956 in Wroclaw/ Breslau, this article questions the existence in Poland of a public space in which the german speaking population could join and confront his voice to that of polish population. It concludes that the newspaper has served as a place of expression of German and Polish vindications, but the political authorities have quickly ended with this German-Polish public space. 